\title{
The xenon hyperpolarization by alexandrite laser spin exchange optical pumping
}

\author{
Alexey Putilov ${ }^{1,2, *}$, Alexandr Antipov ${ }^{1,2}$, and Alexandr Shepelev ${ }^{1}$ \\ ${ }^{1}$ Branch of FSRC "Crystallography and Photonics" RAS, 140700 Shatura, Moscow region, Russia \\ ${ }^{2}$ Vladimir State University named after A. G. and N. G. Stoletovs, 600000 Vladimir, Russia
}

\begin{abstract}
This paper shows the possibility of the use of a solid-state alexandrite laser as a radiation source for the method of spin-exchange optical pumping of noble gases (xenon, krypton). The use of dispersive optical elements in the laser cavity will allows the adjusting of the radiation wavelength exactly in the rubidium spectral absorption lines $794.7 \mathrm{~nm}$ and $780 \mathrm{~nm}$. To obtain a hyperpolarized state of noble gases, it is necessary to excite rubidium atoms for further spin-exchange process with the noble gas nuclei. This fact will allow the increasing of the magnetic resonance imaging contrast in the field of diagnosis of respiratory organs diseases.
\end{abstract}

Magnetic resonance imaging (MRI) is used to diagnose respiratory diseases, as one of the safest and most effective medical methods. Currently, MRI is based on measuring of the electromagnetic response of the hydrogen atoms nuclei, i.e. tomographic images are obtained through the use of the phenomenon of nuclear magnetic resonance (NMR). With this approach, it is difficult to visualize all human organs, such as the lungs and brain. One of the promising ways for the development of MRI is using hyperpolarized noble gases Xe, $\mathrm{Kr}$, which, when inhaled by a person, increase the resolution of this diagnostic method. The nuclear polarization degree of noble gas atoms increases due to spin-exchange optical pumping (SEOP) of alkali metal vapour, which makes it possible to increase the contrast of the obtained NMR images by 5-10 times [1].

This method is based on the phenomenon of spin exchange between the valence electron of an alkali metal atom in the gas phase and the nucleus of a noble gas due to collisions or the formation of Van-der-Waals interactions (Fig. 1). As the alkali metal, Rb is usually used, which has an absorption spectrum in the near infrared region. [2]

$\mathrm{Rb}$ atoms have two transitions, D1 and D2 (Fig. 1b), when the laser is absorbed at wavelengths of $794.7 \mathrm{~nm}$ and $780 \mathrm{~nm}$ [3].

To pump the $\mathrm{Rb}$ atoms - powerful laser diodes and Ti:Sa lasers emitting at a wavelength of $794.7 \mathrm{~nm}$ are used. Ti:Sa lasers are capable of generating narrow-band radiation with a high pulse repetition rate, but they are large, high cost at a low average radiation power in practical applications in SEOP. Powerful laser diodes are compact source of radiation.

\footnotetext{
*Corresponding author: putilov.iplit@ya.ru
} 
a)

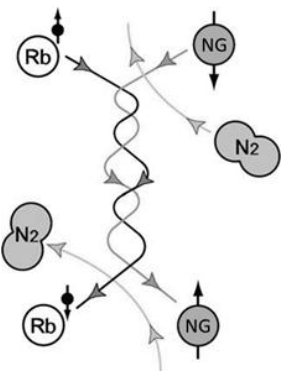

b)

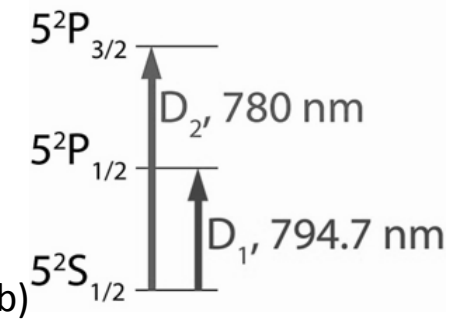

Fig. 1. a) Spin exchange between the valence electron of the $\mathrm{Rb}$ atom and the nucleus of the noble gas $\mathrm{NG}$ atom (for example, 129Xe [2]). b) Excitation transitions of a rubidium electron.

The width of the lasing band is about $2-3 \mathrm{~nm}$, the divergence of the laser diode radiation is extremely large without a special optical system that provides narrowing of the spectrum and the formation of a parallel radiation beam, and the use of high-power laser diodes for efficient pumping of $\mathrm{Rb}$ vapor is not possible. [4]

It is possible to use a solid-state laser based on a synthetic alexandrite crystal, which is a type of chrysoberyl doped with $\mathrm{Cr}^{3+}$ ions as a promising source of laser radiation for SEOP. The emission spectrum of the alexandrite laser lies in the region of $700-850 \mathrm{~nm}$. The high optical and excellent thermomechanical properties of the alexandrite crystal allow to use it as an active element in the development of high power laser technologies operating both in continuous and in pulse-periodic modes $[5,6]$. The use of dispersion elements in the alexandrite laser resonator will allow the tuning of central radiation wavelength from 750 $\mathrm{nm}$ to the $\mathrm{Rb}$ effective pumping wavelength: $780 \mathrm{~nm}$ and/or $794.7 \mathrm{~nm}$ [7]. The construction of a powerful narrowband tunable alexandrite laser will make it possible to create a compact and cheap instrument, which will lead to the development of the SEOP method for hyperpolarizing MRI diagnostics.

This work was supported by the Ministry of Science and Higher Education within the State assignment FSRC «Crystallography and Photonics» RAS.

\section{References}

1. Albert, M. S.,et. al., Nature 370, 199 (1994)

2. T. G. Walker and W. Happer: Spin-exchange optical pumping ... Reviews of Modern Physics, Vol. 69, No. 2 (1997)

3. Steck D. A., Rubidium 85 D Line Data. Oregon Center for Optics and Department of Physics, University of Oregon, 2013.,4 Steck, D. A., Rubidium 87 D Line Data. Oregon Center for Optics and Department of Physics, University of Oregon (2015)

4. Nelson, Chann, and Walker Spin-exchange optical pumping using a frequencynarrowed high power diode laser, Appl. Phys. Lett., Vol. 76, No. 11, 13 March (2000)

5. Achaya Teppitaksak, Ara Minassian, Gabrielle M. Thomas, and Michael J. Damzen, Opt. Express 22, 16386-16392 (2014)

6. Antipov A., Putilov A. TUNABLE ALEXANDRITE LASER Proceedings VII International conference "Modern Nanotechnologies and Nanophotonics for Science and Industry". p. 64-65, (2018)

7. A.Putilov, A. Antipov, A. Shepelev, A. Lotin, S. Arakelyan «Control of the lasing spectrum of Alexandrite laser», Journal of Physics: Conference Series. - 2019 (accepted for publication) 